\title{
The role of estrogen receptor signaling in suppressing the immune response to cancer
}

\author{
James M. Rae ${ }^{1,2}$ and Marc E. Lippman ${ }^{3}$ \\ 'Division of Hematology and Oncology, Department of Internal Medicine, and ${ }^{2}$ Department of Pharmacology, University of Michigan Medical School, Ann Arbor, Michigan, USA. ${ }^{3}$ Georgetown Lombardi \\ Comprehensive Cancer Center, Ceorgetown University, Washington DC, USA
}

\begin{abstract}
Immune checkpoint blockade (ICB) therapies are standard of care for the treatment of many solid tumors. While some patients with cancer experience exceptional and long-term responses, intrinsic and acquired mechanisms of resistance limit the clinical efficacy of ICBs. In addition, ICBs can elicit life-threatening side effects. Alternative options that can increase ICB responses without added toxicities are needed. In this issue of the $J C I$, Chakraborty et al. explored the role of estrogen receptor $\alpha(E R \alpha)$ in modulating ICB activity. Using transcriptomics and preclinical melanoma models, the authors show that ER $\alpha$ signaling in tumor-associated macrophages contributed to an immune-suppressive state within the tumor microenvironment (TME) by promoting $\mathrm{CD}^{+} \mathrm{T}$ cell dysfunction and exhaustion. Further, in murine melanoma models, the addition of fulvestrant, a selective estrogen receptor downregulator (SERD) approved for the treatment of breast cancer, enhanced the antitumor effects of ICB. These results provide a rationale for human trials to test the combination of antiestrogens with ICBs.
\end{abstract}

\section{ER $\alpha$ signaling remodels the tumor microenvironment}

One of the limitations of conventional cancer treatment is the incomplete consideration of interactions between tumor cells with their microenvironment. Most targeted cancer therapies do not address the interplay between cancer cells and their essential host nontumor support cells. Healthy host cells are inherently more genetically stable and could provide a less variable target for emerging therapies. One of the critical factors regulating the tumor microenvironment (TME) may be estrogenic stimulation (Figure 1).

Estrogen receptor $\alpha(\mathrm{ER} \alpha)$ is expressed in multiple cell types, and its activity is involved in multiple aspects of normal human physiology, including the growth and development of female reproductive tissues, bone integrity, cardiovascular and central nervous system functions, normal mammary development, and immune response. Studies suggest that in patients with cancer, men may receive greater benefit from immunotherapy than women and that this phenomenon is related to sex differences in circulating steroid hormones. Specifically, higher circulating estrogen concentrations in women likely promote tumor growth via a cancer cell-extrinsic mechanism by modulating the TME. Results from a 2018 meta-analysis evaluating randomized trials of immune checkpoint blockade (ICB) agents used to treat cancers, including melanoma, non-smallcell lung, renal cell, urothelial, head and neck, gastric, and mesothelioma cancers, found that overall survival rates for men were substantially higher than those for

\section{Related Article: https://doi.org/10.1172/JCI151347}

Conflict of interest: MEL has equity in and receives income from Seattle Genetics Inc.

women (1). This analysis excluded clinical trials of anti-programmed death ligand 1 (anti-PD-L1) drugs, and a more recent meta-analysis found that the patient's sex was not associated with ICB efficacy (2). However, evidence from patients who were not treated with ICBs suggests that high estrogen levels and ER $\alpha$ signaling increase the risk of developing melanoma for women. Conversely, the use of adjuvant antiestrogen therapy in patients with breast cancer is associated with a lower risk of developing secondary melanoma compared with the general population (3). Whether there are true sex differences in responses to ICB therapy remains controversial. However, strong, supportive data suggest that ER $\alpha$ signaling modulates the immune TME, leading to ICB resistance. In addition, given that men also have circulating estrogens, the study in this issue of the JCI by Chakraborty et al. (4) showing that inhibition of ER $\alpha$ signaling can enhance the response to ICB therapy is therefore relevant to cancers in both sexes.

To investigate the role of ER $\alpha$ in modulating the antitumor activity of ICBs, Chakraborty and co-authors analyzed transcriptomic data sets from patients with melanoma to test for correlations between signatures of tumor myeloid cell infiltration and patients' response to ICB therapy. The authors found that the predominant suppressive myeloid cells in the TME were myeloid-derived suppressor cells (MDSCs) and tumor-associated macrophages (TAMs). The MDSC signatures were not predictive of the patient's response; however, a signature for polarized TAMs was associated with the response. Specifically, enrichment of the M1, but not M2, macrophage gene signature was associated with better responses to ICB therapy. Furthermore, the $\mathrm{M} 1 / \mathrm{M} 2$ ratio gene signature was associated with better overall survival in patients with melanoma receiving ICB therapy (4). These results, and studies showing that ER $\alpha$ signaling affects the 


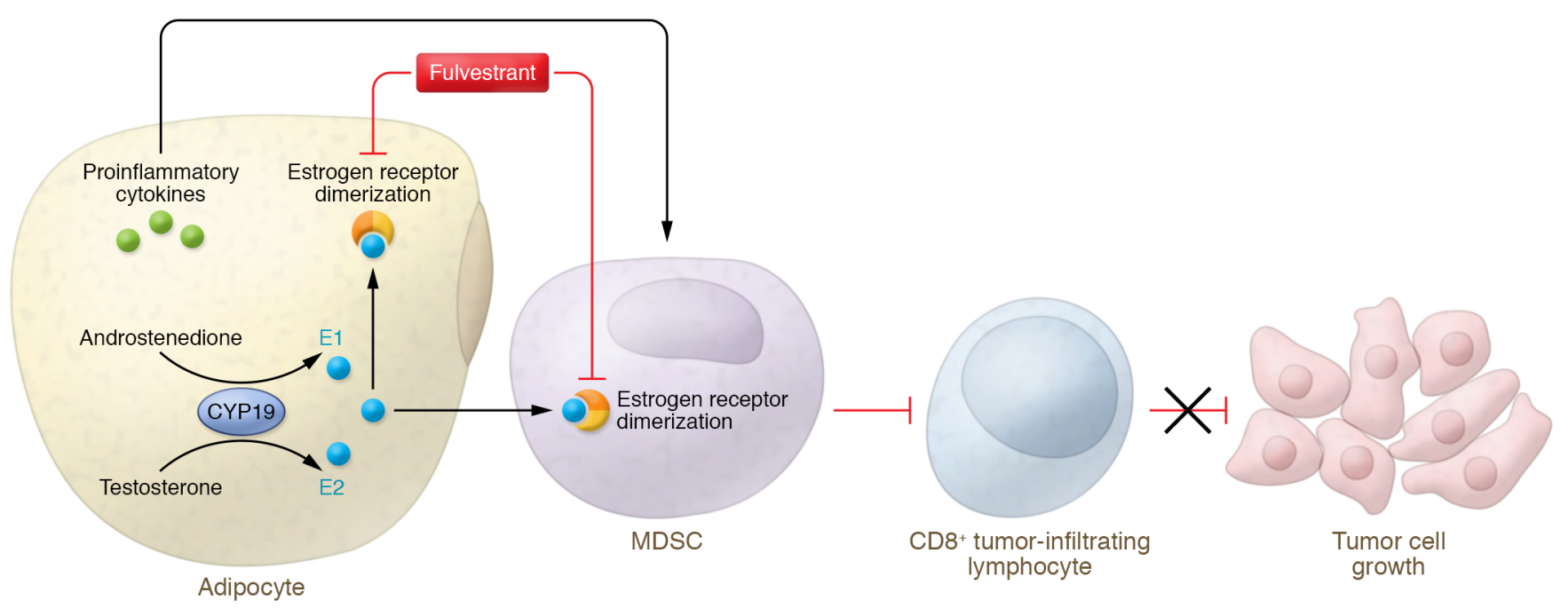

Figure 1. Model for estrogen remodeling of the TME. E1 and E2 signal through the estrogen receptor in MDSCs to promote tumor growth. Estrogen signaling increases the immunosuppressive activities of tumor-infiltrating immune cells, including the suppression of CD8 ${ }^{+} \mathrm{T}$ cells. Treatment with fulvestrant inhibits tumor growth by blocking ER $\alpha$ signaling and increasing the intratumoral macrophage ratio and cytotoxic T cell capabilities. Fulvestrant also blocks estrogen signaling within adipocytes. Adipocytes produce estrogen via the enzyme CYP19 and release cytokines that recruit macrophages and T cells and induce chronic inflammation.

immune system (5), led the authors to hypothesize that ER $\alpha$ modulates the TME, resulting in ICB resistance. They tested this theory using melanoma as a model for ICB activity. First, they showed that $\mathrm{ER} \alpha$ was expressed at negligible levels in the melanoma cells, which established the utility of these models for studying cancer cell-extrinsic actions of ER $\alpha$ signaling. Using three separate syngeneic murine melanoma models, the researchers showed that $17 \beta$-estradiol (E2) treatment in tumor-bearing ovariectomized mice led to increased tumor growth. In addition, E2 treatment in an autochthonous murine model of activated B-Raf ${ }^{\mathrm{V} 600 \mathrm{E}}$ and homozygous deletion of Pten also led to increased tumor growth. RNA-Seq of tumor-infiltrating immune cells isolated from these tumors showed that $\mathrm{E} 2$ treatment resulted in changes in gene expression patterns in TAMs. Additional studies, including depletion of ER $\alpha$ in the mouse myeloid cells led the researchers to conclude that ER $\alpha$ signaling increases the immunosuppressive activities of tumor-infiltrating myeloid cells. In all, the preclinical study results suggest that E2 activation of ER $\alpha$ exerts a direct effect on macrophages to suppress the proliferation and activity of both $\mathrm{CD} 4^{+}$ and $C D 8^{+} \mathrm{T}$ cells (4).

Having established that ER $\alpha$ signaling increased the immunosuppressive activity of tumor-infiltrating myeloid cells, the authors tested whether blocking ER $\alpha$ using fulvestrant, a selective estrogen receptor downregulator (SERD) approved for the treatment of breast cancer, could inhibit tumor growth in vivo. Substantial tumor growth inhibition was observed in all three syngeneic models when fulvestrant was given alone at a dose comparable to that used in patients with breast cancer. These results suggest that fulvestrant treatment alone can inhibit melanoma tumor growth by blocking the effects of ER $\alpha$ signaling and suppressing the immune response (Figure 1). An important next step was to determine whether the combination of fulvestrant with the immune checkpoint inhibitor anti-programmed cell death 1 (anti-PD-1) in both PD-1-sensitive and PD-1-resistant murine melanoma models was superior to single-agent treatment. In both models, the combination outperformed the single agent. Taken together, their in vivo studies indicate that pharmacological targeting of ER $\alpha$ can improve the efficacy of ICBs (4).

It is of some interest to determine the mechanisms of hormonal stimulation responsible for this immunosuppression. A critical issue in evaluating this work involves the choice of estrogens. Recent data have shown that estrone (E1) is not simply a slightly weaker ER $\alpha$ agonist but rather evokes a critically different ER $\alpha$-regulated transcriptome with an emphasis on proinflammatory pathways mediated by NF- $\kappa B$ (6). After menopause, ovarian E2 declines, and estrone (E1), synthesized from adrenal androstenedione by aromatase primarily in fat, dominates (7). In obesity, androstenedione synthesis remains unchanged (8), but its aromatization to $\mathrm{E} 1 \mathrm{in}$ fat increases (9). Consequently, women with obesity have two to four times higher E1 levels, and both obesity and high E1 levels correlate with a greater ER-positive breast cancer risk after menopause (10). Adipocytes mediate inflammation and immunosuppression by activating NF- $\kappa B$ and cytokine induction. Adipose tissue releases cytokines that recruit macrophages and $\mathrm{T}$ cells to induce chronic inflammation (11-13). An expanded pre-adipocyte population produces proinflammatory cytokines, including IL-6, IL-8, and CCL2, that drive pre-adipocyte proliferation (11) and stimulate IL-1 $\beta$ and TNF- $\alpha$ to recruit monocytes and $\mathrm{T}$ cells, thereby perpetuating inflammation (14). Recent experiments in breast cancer models indicate that the tumor-promoting activities of E2 are far less than those of E1 (5), which may explain the mechanism by which receptor inactivation by SERDs decreases the proinflammatory and immunosuppressive environment. This mechanism also opens the possibility of blocking estrogen production (E1 and E2) in the treatment of cancer using an aromatase inhibitor (AI) in 
postmenopausal women, or ovarian suppression therapy in combination with an AI in premenopausal women.

\section{Clinical implications}

ICB therapy has proven effective for the treatment of many solid tumors, but its efficacy in treating breast cancer is modest. Given the results from Chakraborty et al. (4) showing that ER $\alpha$ has cancer cellextrinsic effects that promote tumor growth by inhibiting host immune response, it is interesting to consider how this effect might contribute to breast cancer. While antiestrogens are effective treatments, they do not work in patients with ER-negative breast cancers, and nearly one-third of patients with ER-positive disease receive no benefit. Moreover, all patients with ER-positive metastatic breast cancer (MBC) ultimately become refractory to all known antiestrogens (15). Therefore, several clinical studies have explored the efficacy of single-agent ICB therapy in patients with MBC. For example, in the KEYNOTE-086 study, the response rate to single-agent pembrolizumab in previously treated metastatic triple-negative breast cancer (mTNBC) was $5.3 \%$, but a subset of patients with PD-L1positive tumors and no previous treatment had an objective response rate (ORR) of $21.4 \%$ (16). In a study of single-agent pembrolizumab in PD-L1-positive advanced ER-positive breast cancers, KEYNOTE-028 reported an ORR of $12.0 \%$ (17). Although the rates of response to anti-PD-1/antiPD-L1 monotherapy appear to be modest in both ER-positive MBC and MTNBC, select patients do achieve durable responses. ER-positive breast cancer is thought to be relatively immunologically cold compared with TNBC, largely indicated by low-frequency biomarkers that also predict ICB benefit in the primary disease setting. For example, in primary tumors, HR-positive breast cancer on average has lower levels of $\mathrm{CD}^{+}$tumor-infiltrating lymphocytes, less PD-L1 positivity on tumor cells and immune cells, and a lower somatic mutational burden compared with TNBC (18-20). To date, clinical trials testing ICB for the treatment of breast cancer have been conducted in patients with mTNBC or ER-positive MBC who have become refractory to antiestrogen therapy. Therefore, the combination of ICBs with antiestrogens has yet to be tested and could prove effective in these patients. If estrogens (E1 and E2) exert critical action within the TME and on adipocytes (or MDSCs) to promote the immunosuppressive environment, drugs such as fulvestrant may have a role to play in tumors in which the cancer cells themselves are ER negative, such as TNBC or melanoma.

\section{Acknowledgments}

We would like to thank Erin Cobain for her helpful insights and discussions.

Address correspondence to: James M. Rae, University of Michigan Medical School, 6310 Cancer Center, 1500 E. Medical Center Dr., Ann Arbor, Michigan 48109, USA. Phone: 734.764.1460; Email: jimmyrae@umich.edu.

1. Conforti F, et al. Cancer immunotherapy efficacy and patients' sex: a systematic review and meta-analysis. Lancet Oncol. 2018;19(6):737-746.

2. Wallis CJD, et al. Association of patient sex with efficacy of immune checkpoint inhibitors and overall survival in advanced cancers: a systematic review and meta-analysis. JAMA Oncol. 2019;5(4):529-536.

3. Huber C, et al. Antiestrogen therapy for breast cancer modifies the risk of subsequent cutaneous melanoma. Cancer Prev Res (Phila). 2012;5(1):82-88.

4. Chakraborty B, et al. Inhibition of estrogen signaling in myeloid cells increases tumor immunity in melanoma. J Clin Invest. 2021;131(23): 151347.

5. Khan D, Ahmed SA. The immune system is a natural target for estrogen action: opposing effects of estrogen in two prototypical autoimmune diseases. Front Immunol. 2015;6:635.

6. Qureshi R, et al. The major pre- and postmenopausal estrogens play opposing roles in obesity-driven mammary inflammation and breast cancer development. Cell Metab. 2020;31(6):1154-1172.
7. Siiteri PK. Review of studies on estrogen biosynthesis in the human. Cancer Res. 1982;42(8 suppl):3269s-3273s.

8. Siiteri PK, MacDonald PC. Role of extra-glandular oestrogen in humans endocrinology. In: Greep RO, Astwood EB, eds. Handbook of Physiology, Section 7: Endocrinology (Vol. 2). American Physiological Society; 1973:615-629.

9. Schindler AE, et al. Conversion of androstenedione to estrone by human fat tissue. J Clin Endocrinol Metab. 1972;35(4):627-630.

10. Vincze B, et al. Serum estrone concentration, estrone sulfate/estrone ratio and BMI are associated with human epidermal growth factor receptor 2 and progesterone receptor status in postmenopausal primary breast cancer patients suffering invasive ductal carcinoma. Springerplus. 2015;4:387.

11. Gilbert CA, Slingerland JM. Cytokines, obesity, and cancer: new insights on mechanisms linking obesity to cancer risk and progression. Annu Rev Med. 2013;64(1):45-57.

12. Simpson ER, Brown KA. Minireview: obesity and breast cancer: a tale of inflammation and dysregulated metabolism. Mol Endocrinol. 2013;27(5):715-725.

13. Vona-Davis L, Rose DP. Angiogenesis, adipokines and breast cancer. Cytokine Growth Factor Rev. 2009;20(3):193-201.

14. Catalan V, et al. Adipose tissue immunity and cancer. Front Physiol. 2013;4:275.

15. Burstein HJ, et al. American Society of Clinical Oncology clinical practice guideline: update on adjuvant endocrine therapy for women with hormone receptor-positive breast cancer. J Clin Oncol. 2010;28(23):3784-3796.

16. Adams S, et al. Pembrolizumab monotherapy for previously untreated, PD-L1-positive, metastatic triple-negative breast cancer: cohort B of the phase II KEYNOTE-086 study. Ann Oncol. 2019;30(3):405-411.

17. Rugo HS, et al. Safety and antitumor activity of pembrolizumab in patients with estrogen receptor-positive/human epidermal growth factor receptor 2-negative advanced breast cancer. Clin Cancer Res. 2018;24(12):2804-2811.

18. Cimino-Mathews A, et al. PD-L1 (B7-H1) expression and the immune tumor microenvironment in primary and metastatic breast carcinomas. Hum Pathol. 2016;47(1):52-63.

19. Stanton SE, Disis ML. Clinical significance of tumor-infiltrating lymphocytes in breast cancer. JImmunother Cancer. 2016;4:59.

20. Haricharan S, et al. Somatic mutation load of estrogen receptor-positive breast tumors predicts overall survival: an analysis of genome sequence data. Breast Cancer Res Treat. 2014;146(1):211-220. 